\title{
Acute myocardial infarction during continuous electrocardiographic ST segment recording Possible role of bradycardia and hypotension induced by glyceryl trinitrate
}

\author{
TH VON ARNIM, G AUTENRIETH, H-D BOLTE \\ From the I Department of Medicine, Grosshadern University Hospital, University of Munich, Munich, Federal \\ Republic of Germany
}

SUMMARY A 62 year old man developed an inferior wall myocardial infarction during ambulatory ST segment monitoring. Before the onset of persistent ST segment elevation he had taken several glyceryl trinitrate tablets, which was followed by bradycardia and symptoms of hypotension. Whether a paradoxical reaction to glyceryl trinitrate may have triggered the development of myocardial infarction in this case is uncertain.

Compared with sudden death due to arrhythmia ${ }^{12}$ acute myocardial infarction during ambulatory monitoring has been reported less frequently, probably because ST segment elevation could not be recorded reliably. With the improved low frequency response achieved with frequency modulation and calibrated multichannel recorders these problems have been largely overcome, and continuous electrocardiographic monitoring is increasingly used in patients with ischaemic heart disease. ${ }^{3}$ We report a case in which acute posterior myocardial infarction developed in a patient while he was undergoing continuous ambulatory electrocardiographic monitoring. Bradycardia and symptoms of hypotension, both possibly induced by glyceryl trinitrate, immediately preceded the development of permanently elevated ST segments.

\section{Case report}

A 62 year old man was admitted to our hospital for coronary angiography because of stable angina pectoris. He had a history of transient cerebral ischaemia, anterior myocardial infarction eight months earlier, and femoral artery embolism. Four months before admission his heart was of normal size on a chest radiograph and $M$ mode echocardiography. Risk fac-

Requests for reprints to Dr Thomas von Arnim, Medizinische Klinik I, Klinikum Grosshadern der Universität Munchen, Marchioninistrasse 15, D-8000 Munich 70, FRG. tors were smoking ( 20 cigarettes per day) and hypertension for more than 10 years. The electrocardiogram on admission showed a previous anterior myocardial infarction (Fig. 1, day 0). The patient was without symptoms when continuous electrocardiographic monitoring (Oxford Medilog 2-FM) was started. Two bipolar chest leads CM5, channel 1, and CC5, channel 2, were recorded giving traces resembling leads V 5 and III. ${ }^{4}$ Late the following evening the patient had several episodes of chest pain which responded to oral glyceryl trinitrate $(0.8 \mathrm{mg})$. During these episodes there was deepening of the ST segment depression in lead CM5 (Fig. 2, 21.56 hours). At 02.30 the patient noticed further chest pain and took two more glyceryl trinitrate tablets but felt an "inverse" effect. The pain did not subside, and he felt dizzy and noticed blurred vision. At that time the electrocardiogram showed a pronounced sinus bradycardia; the heart rate fell from 90 beats/min at 02.24 to 40 beats $/ \mathrm{min}$ at 02.35 returning to 80 beats/ $\mathrm{min}$ at 02.38 . Lead CC5 in channel 2 showed pronounced ST segment elevation, which also resolved at 02.38 (Fig. 2). From 02.55 onwards ST segment elevation developed again in the posterior lead and also in the anterior lead, where it was less pronounced. ST segment elevation persisted from that time onwards; intermittent first and second degree atrioventricular block occurred and heart rate increased to 112 beats/min at 07.00 . The patient did not summon assistance until after 04.30 , when he was immediately taken to the coronary care unit. The 12 lead electro- 


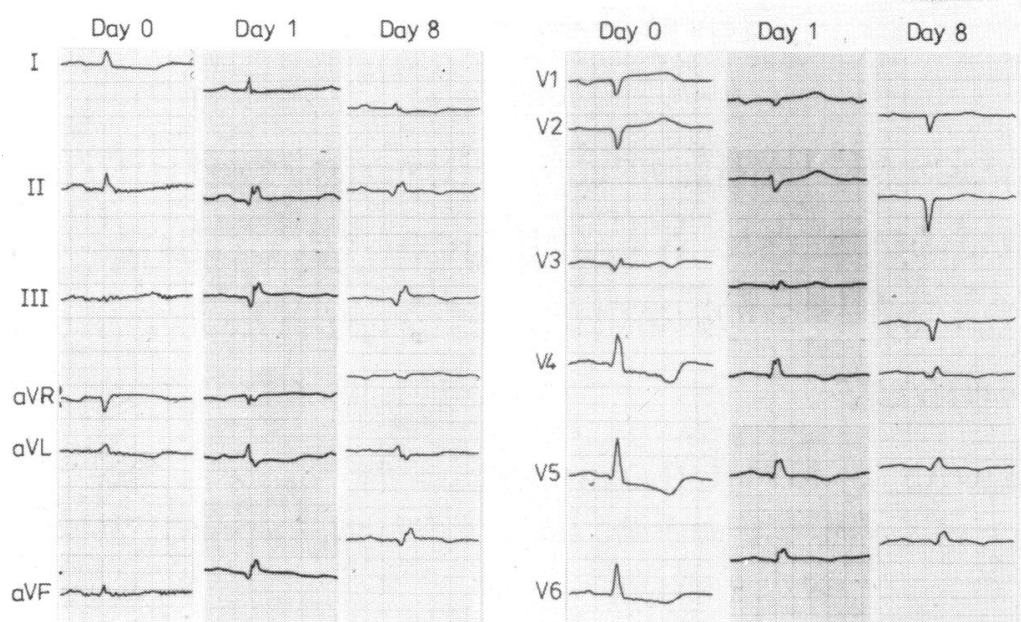

Fig. 112 lead electrocardiograms on the days before and after the acute myocardial infarction. Twenty four hour electrocardiographic monitoring took place from day 0 to day 1. Note the pre-existing signs of anterior myocardial infarction and the new signs of posterolateral infarction appearing on day 1 . The patient had an ejection fraction of $25 \%$ (day 8) on cross sectional echocardiography and severe congestive heart failure.

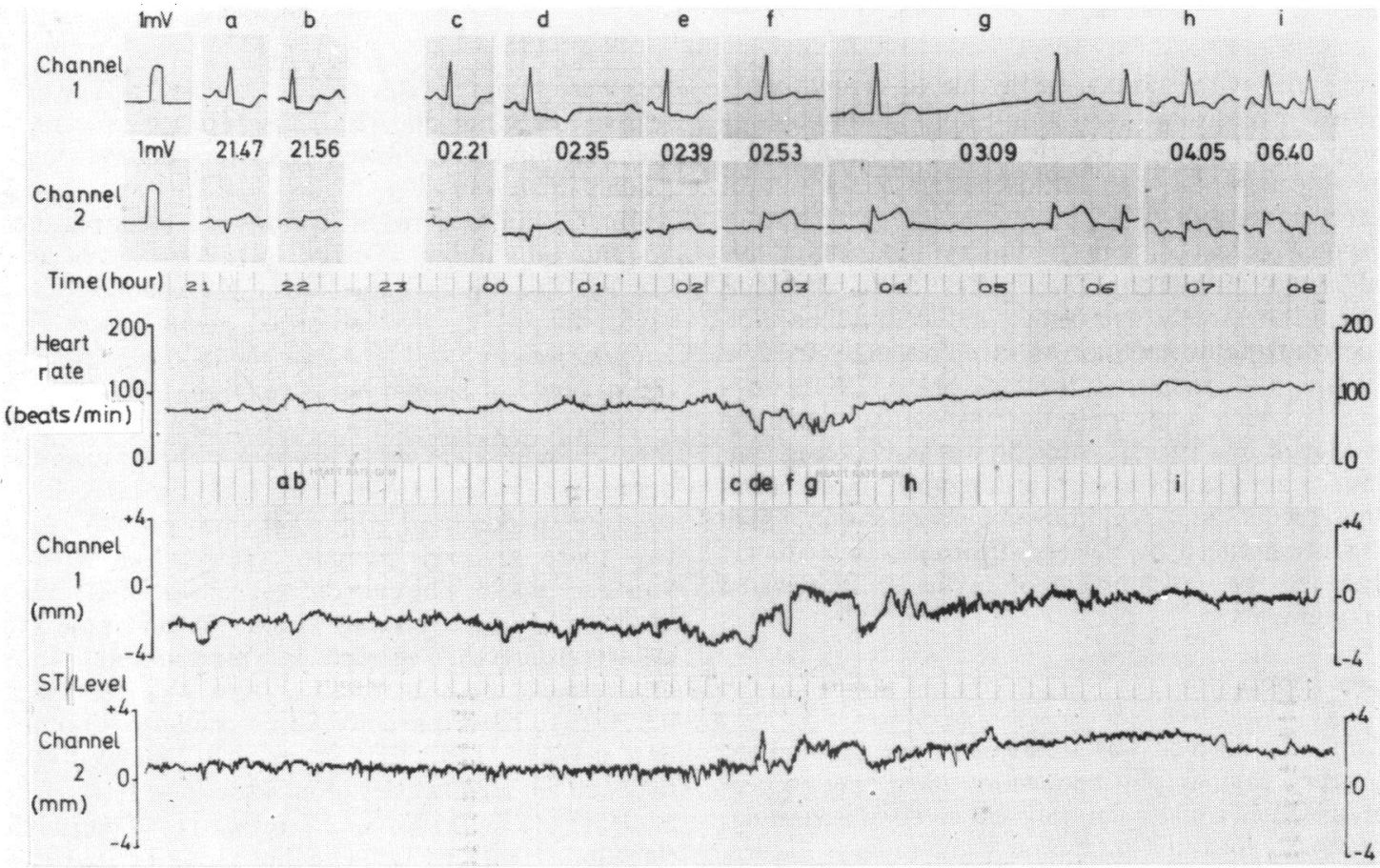

Fig. 2 Selected electrocardiographic tracings and trend recordings of heart rate and ST segment levels. Two bipolar leads were recorded: channel 1 resembles V5 and channel 2 resembles lead III. ST level (mm) is the vertical distance of a point selected on the ST segment from a reference point on the $P Q$ segment. Upper tracings show the calibration (10 mom for $1 \mathrm{mV}$ ) and selected examples from the times indicated $(a-i) .(a-b)$ - the patient experienced angina, which was responsive to glyceryl trinitrate (note the heart rate increase and ST segment depression on channel 1 at 21.56); (c-e-the patient experienced angina, which was not relieved by $1.6 \mathrm{mg}$ glyceryl trinitrate, and dizainess, and blurred vision (note the drop in heart rate from 90 to 40 beats /min; $(f-i) \longrightarrow S T$ segment elevation in both channels; the pain persisted but the patient sumemoned assistance only at 04.30 (note second degree atrioventricular block at (g) (trend 03.00 to 03.30) and increased heart rate due to congestive heart failure at (i)). 
cardiogram showed posterolateral myocardial infarction (Fig. 1, day 1). Serum creatine kinase activity rose to $1310 \mathrm{U} / \mathrm{l}$ at 20.00 hours on that day. He later developed severe congestive heart failure but no further chest pain. He was discharged on the twenty sixth day.

\section{Discussion}

The development of posterolateral acute myocardial infarction could be examined closely in this case. The intake of $1.6 \mathrm{mg}$ glyceryl trinitrate was followed by bradycardia, as recorded and timed on the tape, and by symptoms of hypotension, as related by the patient. The time course of these events-preceding the development of electrocardiographic evidence of permanent ischaemia-makes a reaction to glyceryl trinitrate seem a more likely explanation for the bradycardia and hypotension than a Bezold-Jarisch reflex early in the course of myocardial infarction of the inferior wall. Although this latter possibility cannot be completely ruled out it raises the question of a causative role for the syndrome of glyceryl trinitrate induced bradycardia and hypotension 56 in the myocardial infarction which developed thereafter. Paradoxical reactions of coronary arteries to glyceryl trinitrate have been reported ${ }^{78}$ and may be a reason for this rare syndrome. The routine use of glyceryl trinitrate before coronary angiography has been debated.910 In our case paradoxical coronary vasospasm may have led to myocardial infarction, ${ }^{11}$ or, alternatively, coronary thrombosis may have been triggered by low flow due to reduced coronary perfusion pressure. Continuous ST segment monitoring during the transition from unstable angina pectoris to acute myocardial infarction may in future offer more insights into the time course and correlation of pain, electrocardiographic changes, and arrhythmias. Knowledge of this time course in many cases is impor- tant for the planning of therapeutic interventions-for example, efforts to recanalise coronary arteries occluded by a thrombus.

\section{References}

1 Møller M, Straede-Nielsen J, Oxhøj H. Sudden death during ambulatory electrocardiography [Letter]. Lancet 1981; ii: 1117-8.

2 Lahiri A, Balasubramanian V, Raftery EB. Sudden death during ambulatory monitoring. $\mathrm{Br}$ Med $\mathcal{F} 1979$; i: 16768.

3 Johnson SM, Mauritson DR, Winniford MD, et al. Continuous electrocardiographic monitoring in patients with unstable angina pectoris: identification of high-risk subgroup with severe coronary disease, variant angina, and/ or impaired early prognosis. Am Heart f 1982; 103: 4-12.

4 Balasubramanian V, Lahiri A, Green HL, Stott FD, Raftery EB. Ambulatory ST-segment monitoring: problems, pitfalls, solutions, and clinical application. Br Heart $\mathcal{f}$ 1980; 44: 419-25.

5 Nemerovski M, Shah PK. Syndrome of severe bradycardia and hypotension following sublingual nitroglycerin administration. Cardiology 1981; 67: 180-9.

6 Come PC, Pitt B. Nitroglycerin-induced severe hypotension and bradycardia in patients with acute myocardial infarction. Circulation 1976; 54: 624-8.

7 Feldman RL, Pepine CJ, Conti CR. Unusual vasomotor coronary arterial responses after nitroglycerin. $A m \mathcal{F}$ Cardiol 1978; 42: 517-9.

8 Bove AA, Vlietstra RE. Paradoxical angina after nitroglycerin-another variant [Letter]. $N$ Engl f Med 1982; 306: 484-5.

9 Coskey RL. Glyceryl trinitrate before coronary angiography [Letter]. Br Heart $\mathcal{F}$ 1979; 42: 617.

10 Feldman RL, Pepine CJ, Curry RC, Conti CR. Glyceryl trinitrate before coronary angiography [Letter]. Br Heart f 1979; 42: 617-8.

11 Maseri A, L'Abbate A, Baroldi G, et al. Coronary vasospasm as a possible cause of myocardial infarction. $N$ Engl $\mathcal{F}$ Med 1978; 299: 1271-7. 